Case Report

\title{
First Schistosomal Cholecystitis Complicated by Cholangitis and Liver Abscess: Case Report and Review of Literature
}

\author{
Ali Toffaha $\mathbb{D}^{1},{ }^{1}$ Samir Al Hyassat, ${ }^{2}$ Walid Elmoghazy $\mathbb{D}^{1,3}$ Hatem Khalaf $\mathbb{D}^{1,4}$ \\ and Ahmed Elaffandi $\mathbb{D}^{1,5}$ \\ ${ }^{1}$ Department of Surgery, Hamad General Hospital, Doha, Qatar \\ ${ }^{2}$ Department of Laboratory Medicine and Pathology, Hamad General Hospital, Doha, Qatar \\ ${ }^{3}$ Department of Surgery, Sohag University, Cairo, Egypt \\ ${ }^{4}$ Department of Surgery, College of Medicine, Qatar University, Qatar \\ ${ }^{5}$ Department of Surgical Oncology, National Cancer Institute, Cairo, Egypt
}

Correspondence should be addressed to Ali Toffaha; atoffaha2@gmail.com

Received 10 April 2021; Accepted 29 June 2021; Published 13 July 2021

Academic Editor: Eric Bergeron

Copyright (c) 2021 Ali Toffaha et al. This is an open access article distributed under the Creative Commons Attribution License, which permits unrestricted use, distribution, and reproduction in any medium, provided the original work is properly cited.

\begin{abstract}
Schistosomiasis is one of the most prevalent parasitic infections in the developing world. When it affects the gastrointestinal system specifically the liver, it causes periportal fibrosis followed by cirrhosis. Cholecystitis however is a rare presentation, and associated liver abscess has certainly never been reported to date. We report a case of acute cholecystitis complicated by cholangitis and liver abscess in a 46-year-old man. After complex course of treatment, he had laparoscopic cholecystectomy, and the histology report confirmed schistosomiasis. Gallbladder schistosomiasis is an uncommon disease that is associated with dense fibrotic changes that strongly mimics xanthogranulomatous cholecystitis. Liver abscess may occur during the disease evolution especially in patient originating from endemic backgrounds. We present the case and a comprehensive literature review.
\end{abstract}

\section{Introduction}

Parasitic infections remain a problem in the developing countries [1]. Schistosomiasis is responsible for more than 200 thousands deaths yearly [1]. Schistosomiasis may present acutely as febrile illness [2] or more commonly in chronic form due to eggs that are trapped in the tissues during the peri-vesical or peri-intestinal migration or after embolization in the liver, spleen, lungs, or cerebrospinal system [3]. Chronic lesions in these tissues are usually characterized by chronic inflammation and fibrosis which gives rise to the clinical manifestation of the disease (i.e., cirrhosis for hepatic involvement, chronic cystitis, and fibrosis for urinary involvement) [1].

In spite the fact of the high frequency of hepatic involvement particularly by Schistosoma mansoni, schistosomiasis of the gallbladder (GB) is remarkably uncommon [4]. About twelve cases have been retrieved from the literature. However, none of them was associated with liver abscess or cholangitis. A literature review in a comprehensive approach was carried out (Table 1). We report this case in line with the updated consensus-based surgical case report (SCARE) guidelines [5].

\section{Case Presentation}

A 46-year-old Egyptian man presented to the emergency department with one-day history of epigastric and right upper abdominal pain, associated with nausea and vomiting. $\mathrm{He}$ denied any other associated gastrointestinal or urologic symptoms. Apart from being type 1 diabetic, he declared no other significant past medical history.

The patient presented in good shape, and there was no hemodynamic instability. He showed epigastric tenderness with no signs of peritonism with the rest of the abdomen unremarkable. White blood cell count (WBC) was $17.4 \mathrm{~K} / \mathrm{uL}(4-10 \mathrm{~K} / \mathrm{uL})$. Liver function tests (LFTs) were abnormal: total bilirubin $47 \mathrm{umol} / \mathrm{L}(0-21 \mathrm{umol} / \mathrm{L})$, direct bilirubin $35 \mathrm{umol} / \mathrm{L}$ (0-3 umol/L), ALT $156 \mathrm{U} / \mathrm{L}$ (0-40 U/L), and 


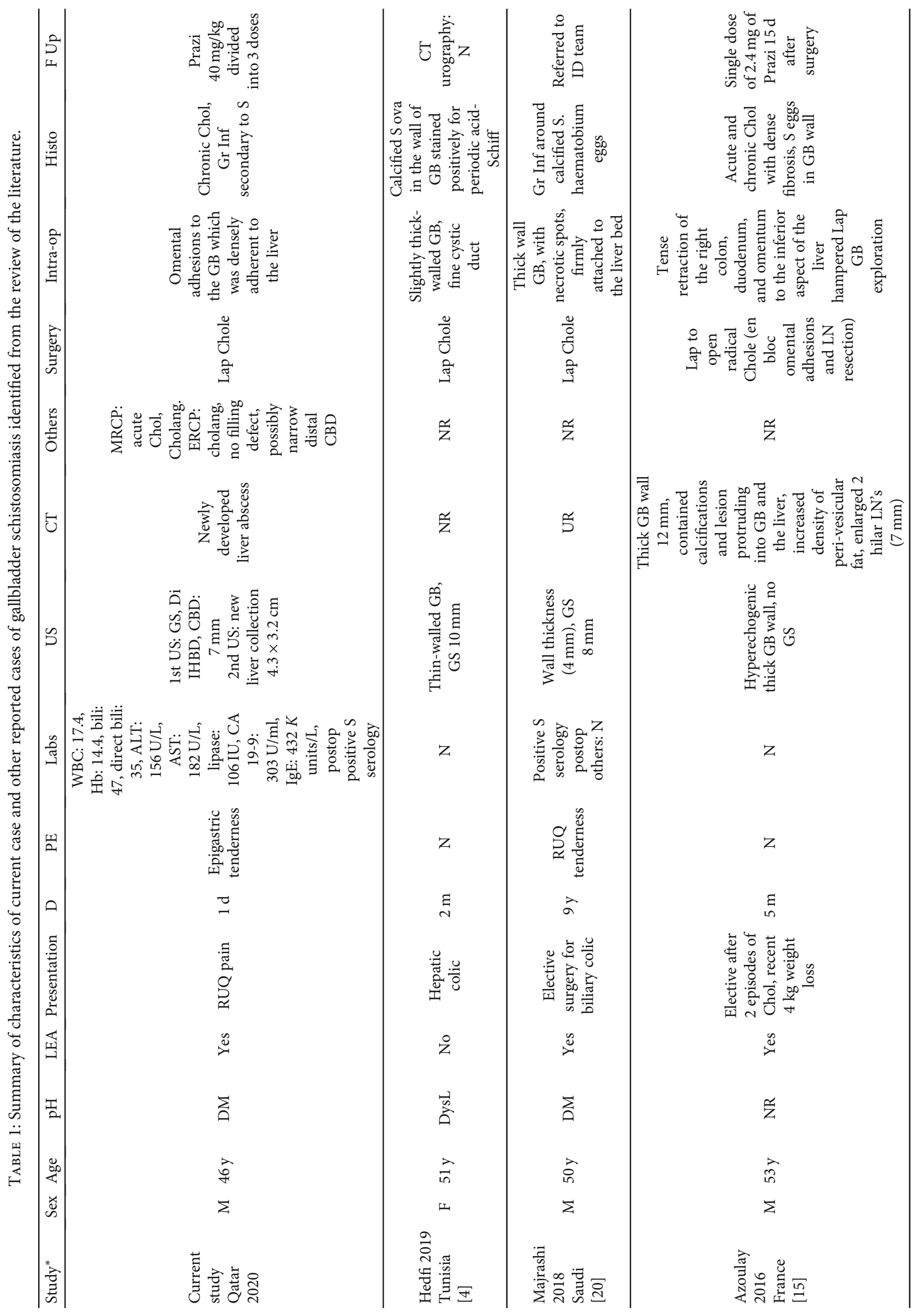




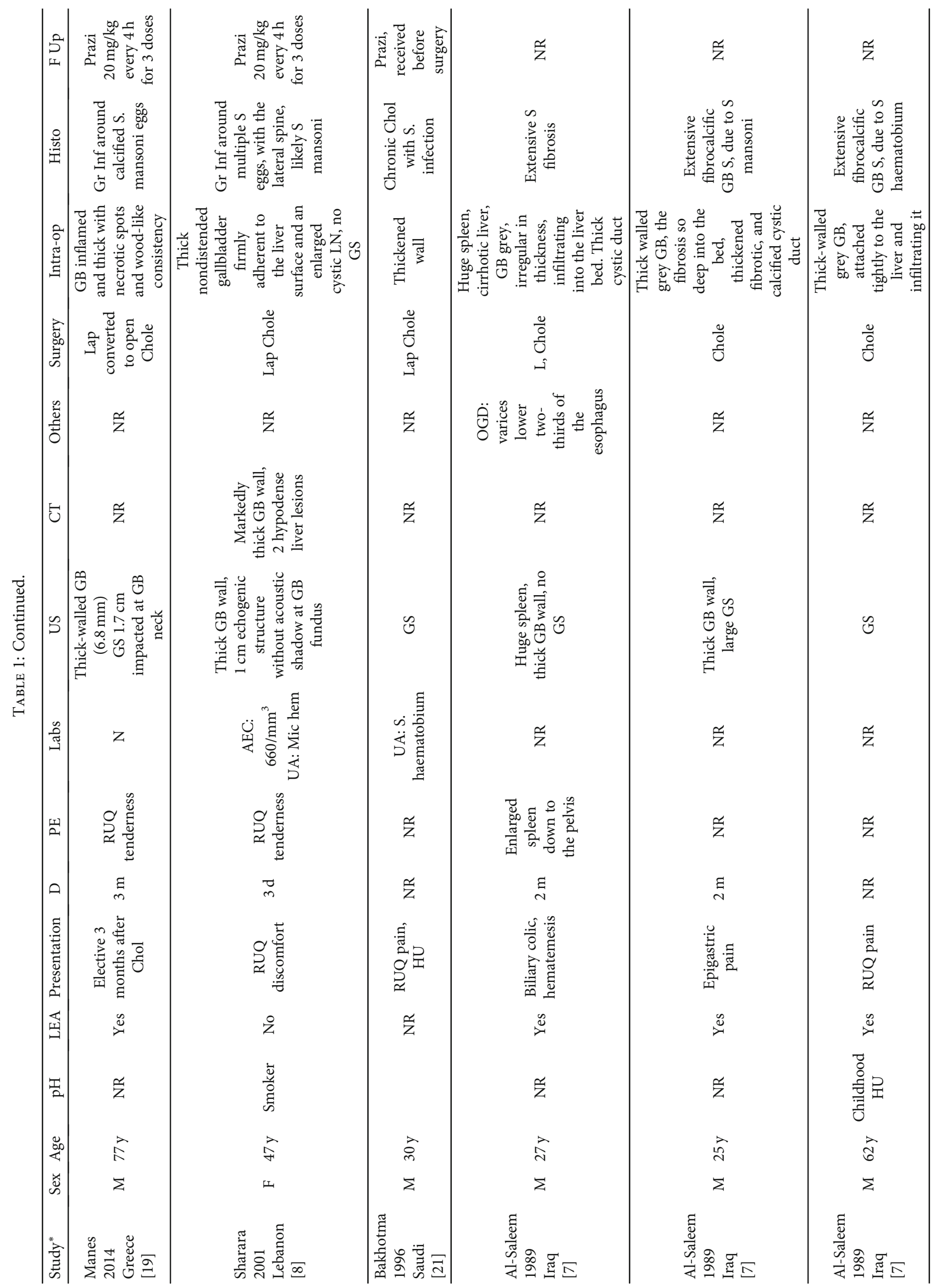




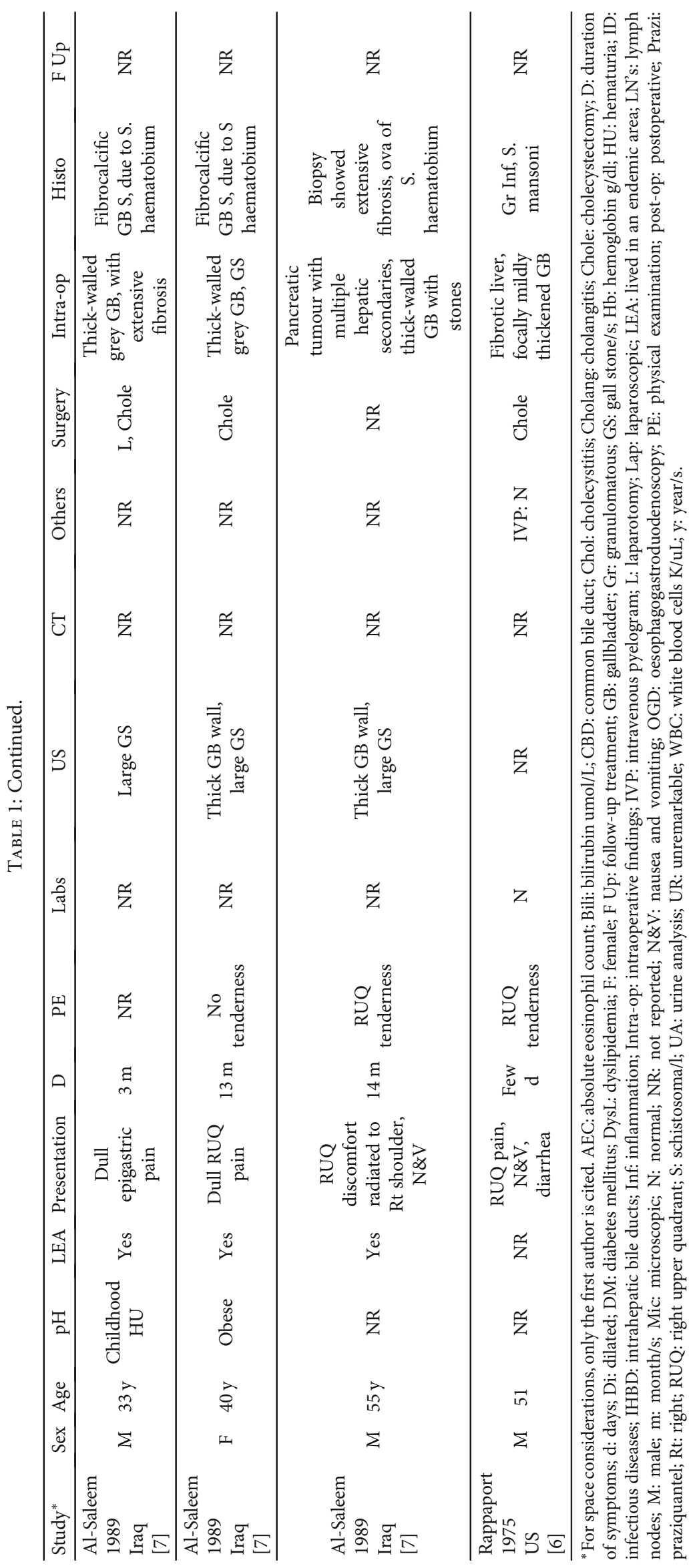


AST $182 \mathrm{U} / \mathrm{L}(0-41 \mathrm{U} / \mathrm{L})$. Other results showed hemoglobin $14.4 \mathrm{~g} / \mathrm{dL}(13-17 \mathrm{~g} / \mathrm{dL})$, platelets $190 \mathrm{~K} / \mathrm{uL}(150-400 \mathrm{~K} / \mathrm{uL})$, lipase $106 \mathrm{U} / \mathrm{L}(13-60 \mathrm{U} / \mathrm{L}), \mathrm{CRP}<5 \mathrm{mg} / \mathrm{L}(0-5 \mathrm{mg} / \mathrm{L}), \mathrm{CA}$ 19-9 $303 \mathrm{U} / \mathrm{ml}(0-27 \mathrm{U} / \mathrm{ml})$, and $\operatorname{IgE} 432 \mathrm{Ku} / \mathrm{L}(0-114$ $\mathrm{Ku} / \mathrm{L})$. IgA and IgG4 were normal. Abdominal ultrasound (US) showed multiple GB stones with intrahepatic biliary dilatation and prominent common bile duct (CBD) measuring $7 \mathrm{~mm}$. The rest of the examination showed no signs of acute cholecystitis and no bile duct stones. The patient was admitted as a case of obstructive jaundice for further work up. He later developed signs of sepsis (tachycardia and fever) for which blood cultures were taken. He was started on intravenous ceftriaxone and metronidazole. Endoscopic retrograde cholangiopancreatography (ERCP) showed purulent bile immediately following cannulation and failed to show any filling defects. Sphincterotomy and CBD stenting were done. ERCP procedure was not extraordinary in difficulty to suspect ampullary fibrosis or deformation. Magnetic Resonance Cholangiopancreatography (MRCP) later showed distended GB containing sludge and tiny stones, hyperenhancement of both GB and CBD walls, and mildly thickened GB wall in addition to pericholecystic edema and fat stranding, consistent with acute cholecystitis and cholangitis. There were no CBD stones nor thickening of the CBD wall. After stenting, the patient was kept on piperacillin/tazobactam. He improved clinically, and both his LFTs and inflammatory markers were trending down till the 4th day post-ERCP when he started to spike fever again. Septic work up was repeated, and endoscopic ultrasound (EUS) was done. This exam showed the stent in place. Both US and computed tomography (CT) showed a new lesion $5 \times 4 \times 5 \mathrm{~cm}$ in the segment IVb of the liver in continuation with the GB fundus (Figure 1). The lesion was compatible with a newly developed liver abscess. A percutaneous aspiration was carried out under US guidance, during which $100 \mathrm{ml}$ of pus was aspirated and sent for microbiology/culture. During the aspiration, the GB was noticed to be deflating which pointed towards continuity between the liver abscess and the GB. Cultures grew Klebsiella oxytoca and Escherichia coli. The patient responded well after aspiration and antibiotic therapy and showed improved inflammatory markers. He was discharged the next day on oral antibiotics with close clinic follow-up to arrange for interval cholecystectomy after ERCP and stent removal.

During follow-up visits, the patient was asymptomatic. ERCP was done 5 weeks after discharge and showed no filling defects in the CBD. The stent was removed. The patient travelled and was lost to follow-up for 5 months. When he came back, an MRCP showed complete resolution of the liver abscess. Multiple gall stones in GB were still demonstrated. He was booked for elective laparoscopic cholecystectomy. Intraoperative findings showed omental adhesions to the GB. The GB was also densely adherent to the liver. Despite the difficult dissection, the procedure was managed laparoscopically. Patient's postoperative course was unremarkable, and he was discharged next day after surgery. Histopathology of the gallbladder showed chronic cholecystitis, with granulomatous inflammation secondary to schistosomiasis (Figure 2).

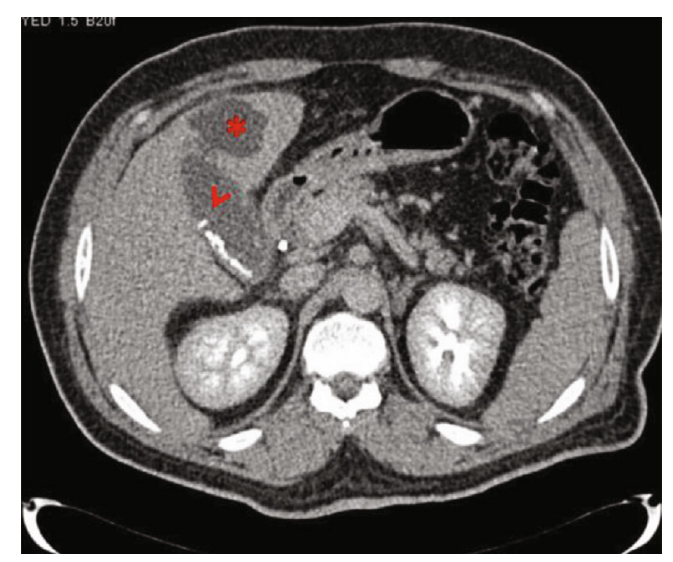

Figure 1: Abdominal CT scan showing gallbladder containing stones (arrow), with nearby segment IV b abscess (asterisk).

\section{Discussion}

The first case of gallbladder schistosomiasis (GBS) was reported in 1975, and since then, speculations were made regarding possible pathogenesis [6]. Fourteen cases of GBS have been reported; however, none of them presented with associated complications. Few theories evolved on how schistosomiasis can cause cholecystitis. Some speculated that the fibrosis of the cystic duct, like what is seen in the ureters of patients with urinary schistosomiasis, causing a stenosis which can contribute to bile stasis and formation of stones in the gallbladder [7]. Others suggested that granulomatous inflammation in the gallbladder's wall makes it prone for stone formation [8].

The risk factor for contracting schistosomal infection is the contact of its larval form with the skin through contaminated water in endemic areas [4]. Most of the reported cases (Table 1) have been living at one stage in their life in an endemic area. Our reported case used to live in Egypt that is a well-known endemic area before moving abroad.

Clinical presentation is variable according to the involved organ. Infestation of urinary tract may lead to hematuria, fibrosis, and obstructive uropathy that may lead to parenchymal renal damage [1]. When it involves the liver, early inflammatory hepatic schistosomiasis happens in reaction to schistosomal eggs trapped in the presinusoidal periportal spaces of the liver. It then lead to typical features of sharpedged enlargement of the liver nodular splenomegaly [1]. Intestinal involvement leads to diarrhea mostly due to mucosal granulomatous inflammation, pseudopolyposis, and microulcerations [1]. Reported symptoms of GBS are usually similar to other gallbladder diseases, including right upper quadrant pain that is sometimes associated with nausea and vomiting [7]. Abdominal examination shows right upper abdominal tenderness especially if the patient is having active cholecystitis (Table 1). The reported case first presented to the emergency with right upper quadrant abdominal pain. His disease progression was completely unique after GBS as he developed septic features due to cholangitis and associated liver abscess. This is, to our knowledge, the first reported case of cholecystitis with a liver abscess in a patient with 


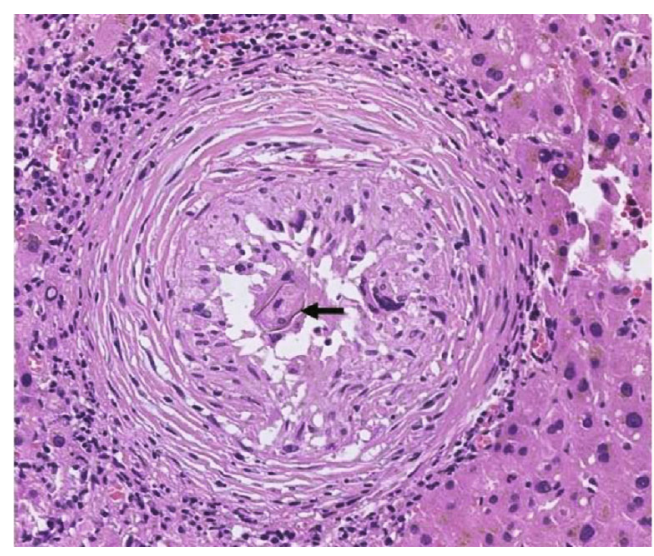

Figure 2: Microscopic image of gallbladder wall showing Schistosoma parasite within noncaseating granuloma (magnification $\mathrm{HE} \times 20$ ).

schistosomiasis. This situation may however be as well secondary to a typical gallstone cholecystitis.

Cholangitis is mostly caused by ascending bacterial infection due to obstruction [9]. Causes of obstruction are variable (benign and malignant), the most common of which are biliary stones that usually slip from the gallbladder [9]. In our case, no stones were identified in the CBD by ERCP or MRCP; however, slipped stone to duodenum cannot be excluded as a probable cause. Another speculation is related to schistosomiasis pathogenesis leading to fibrosis and stricture of the CBD [10]. Despite early fibrotic changes of the CBD and/or ampulla being a possible cause in our case, however, we did not have any imaging evidence indicating gross CBD wall thickening or ampullary fibrosis.

Liver abscess has already been described as a complication of liver schistosomiasis [11]. However, it has not been yet reported as a part of the GBS disease progress (Table 1). Many speculations have been suggested to explain the link between schistosomiasis and liver abscesses. Bacteria tends to bind to laminin, fibronectin, and type IV collagen, which are plentiful in the active schistosomal granuloma. Moreover, the development and degradation of extracellular matrix of the granuloma may play a role on abscess formation [11]. Additionally, deposition of eggs was found to inhibit $\mathrm{T}$ cell response, so the usual immune response to bacteria can be affected [12]. Despite the mentioned speculations for liver abscess pathogenesis, cholecystitis and cholangitis itself can be responsible for this complication, as it is well reported cause for liver abscess whether concomitantly or remotely after resolution of cholecystitis [13]. Acute cholangitis and ERCP instrumentation are among other possible causes for liver abscess [14].

The imaging modality of choice for gallbladder disease is abdominal ultrasound, but it has no specific signs to indicate GBS [4]. In our case, the initial US showed gallstones which is similar to most of the reported cases seen in our review (Table 1). No specific blood tests are available for GBS. Only two cases showed positive serology after surgery (Table 1).

For GBS, the treatment remains surgical, usually by laparoscopic cholecystectomy [4]. At surgery, the gallbladder mimics cancer or xanthogranulomatous cholecystitis [15]. As per review, most cases showed irregularly thick and fibrosed gallbladder wall infiltrating into the liver at its bed alongside adhesions to the omentum and nearby bowel (Table 1). In the case presented, the fibrosis encountered in the liver bed could be secondary to schistosoma infestation, healing process secondary to the cholecystitis, or probably a combination of both. For timing of surgery, we preferred to proceed with an elective cholecystectomy after the patient passed the acute stage of disease and treatment for obstructive jaundice, cholangitis, and liver abscess. Elective cholecystectomy after cholangitis treatment is a feasible option as per Tokyo guidelines [16]. Other studies showed that drainage followed by delayed surgery is an acceptable treatment for cholecystitis concomitant with liver abscess $[17,18]$.

Moreover, four patients received postoperative complementary medical treatment which was praziquantel $[8,15$, 19]. Similar to others, our patient received postoperative praziquantel.

The specimen pathology usually reveals a lymphocytic infiltrate; schistosomal eggs can be found in any layer of the gallbladder wall causing fibrocalcific reaction; most of the cases showed granulomatous inflammation surrounding the schistosomal eggs (Table 1).

\section{Conclusions}

GBS might be considered preoperatively in patients who lived in an endemic area and developed symptoms suggestive of gallbladder disease. This is the first case that report a liver abscess in a patient with cholecystitis with a gallbladder infested by Schistosoma. However, a majority of cholecystitis in patients with schistosomiasis involve the presence of gallstones. This condition carries the same possible complications and should be managed in the same way as usual cholecystitis. Surgeons must however expect a more difficult dissection during operation.

\section{Ethical Approval}

The protocol for this research project has been approved by a suitably constituted Ethics Committee of the institution, and it conforms to the provisions of the Declaration of Helsinki. Medical research center committee of Hamad Medical Corporation, approval No. (MRC-04-20-912).

\section{Consent}

Written informed consent was obtained from the patient for publication of this case report and accompanying images. A copy of the written consent is available on request.

\section{Conflicts of Interest}

The authors declare no conflict of interests for this article. 


\section{References}

[1] B. Gryseels, K. Polman, J. Clerinx, and L. Kestens, "Human schistosomiasis," The Lancet, vol. 368, no. 9541, pp. 11061118, 2006.

[2] E. Bottieau, J. Clerinx, M. R. de Vega et al., "Imported Katayama fever: clinical and biological features at presentation and during treatment," The Journal of Infection, vol. 52, no. 5, pp. 339-345, 2006.

[3] A. W. Cheever, K. F. Hoffmann, and T. A. Wynn, "Immunopathology of schistosomiasis mansoni in mice and men," Immunology Today, vol. 21, no. 9, pp. 465-466, 2000.

[4] M. Hedfi, M. Debaibi, S. Ben Iahouel, and A. Chouchen, "Gallbladder schistosomiasis: rare but possible, a case report and review of the literature," The Pan African Medical Journal, vol. 32, 2019.

[5] R. A. Agha, M. R. Borrelli, R. Farwana et al., "The SCARE 2018 statement: updating consensus Surgical CAse REport (SCARE) guidelines," International Journal of Surgery, vol. 60, pp. 132-136, 2018.

[6] I. Rappaport, J. Albukerk, and I. Schneider, "Schistosomal cholecystitis," Archives of Pathology, vol. 99, no. 4, pp. 227-228, 1975.

[7] T. Al-Saleem and T. Al-Janabi, "Schistosomal cholecystitis: report of six cases," Annals of the Royal College of Surgeons of England, vol. 71, pp. 366-369, 1989.

[8] A. I. Sharara, G. Abi-Saad, M. Haddad, A. Mansour, and A. Tawil, "Acute granulomatous schistosomal cholecystitis," European Journal of Gastroenterology \& Hepatology, vol. 13, no. 8, pp. 1001-1003, 2001.

[9] D. Lan Cheong Wah, C. Christophi, and V. Muralidharan, "Acute cholangitis: current concepts," ANZ Journal of Surgery, vol. 87, no. 7-8, pp. 554-559, 2017.

[10] C. H. McCrann and J. L. Boyer, "Small-duct sclerosing cholangitis associated with Schistosoma mansoni," Hepatology, vol. 53, no. 2, pp. 712-713, 2011.

[11] L. Z. Goldani, R. P. dos Santos, and A. M. Sugar, "Pyogenic liver abscess in patients with schistosomiasis mansoni," Transactions of the Royal Society of Tropical Medicine and Hygiene, vol. 99, no. 12, pp. 932-936, 2005.

[12] J. M. Grzych, E. Pearce, A. Cheever et al., "Egg deposition is the major stimulus for the production of Th2 cytokines in murine schistosomiasis mansoni," Journal of Immunology, vol. 146, pp. 1322-1327, 1991.

[13] G. Zerman, M. Bonfiglio, G. Borzellino et al., "Liver abscess due to acute cholecystitis. Report of five cases," Chirurgia italiana, vol. 55, no. 2, pp. 195-198, 2003.

[14] T. Yamada, K. Murakami, K. Tsuchida et al., "Ascending cholangitis as a cause of pyogenic liver abscesses complicated by a gastric submucosal abscess and fistula," Journal of Clinical Gastroenterology, vol. 30, no. 3, pp. 317-320, 2000.

[15] D. Azoulay, M. Djabbari, and J. Calderaro, "An unusual cause of cholecystitis," Gastroenterology, vol. 150, no. 5, pp. e3-e4, 2016.

[16] F. Miura, K. Okamoto, T. Takada et al., “Tokyo Guidelines 2018: initial management of acute biliary infection and flowchart for acute cholangitis," Journal of Hepato-BiliaryPancreatic Sciences, vol. 25, no. 1, pp. 31-40, 2018.

[17] D. Santos Gabriel, V. Eduardo, O. Pertusso Eduardo, C. Author, and P. Eduardo, "Cholecystitis and synchronous liver abscess: percutaneous treatment results," Acta Scientific Gastrointestinal Disorders, vol. 2, no. 9, pp. 16-21, 2019.

[18] R. Costi, A. Le Bian, F. Cauchy et al., "Synchronous pyogenic liver abscess and acute cholecystitis: how to recognize it and what to do (emergency cholecystostomy followed by delayed laparoscopic cholecystectomy)," Surgical Endoscopy, vol. 26, no. 1, pp. 205-213, 2012.

[19] K. Manes, K. Chatzimargaritis, D. Apessou, V. Papastergiou, and C. Dervenis, "Granulomatous cholecystitis in a patient with schistosoma mansoni infection: a case report," International Journal of Case Reports and Images, vol. 5, no. 6, p. 439, 2014.

[20] S. A. Majrashi and O. M. Al Amoodi, "Schistosomiasis as a cause of acute cholecystitis," Saudi Medical Journal, vol. 39, no. 7, pp. 725-728, 2018.

[21] M. A. Bakhotmah, "Gallbladder bilharziasis," HPB Surgery, vol. 9, no. 3, pp. 175-177, 1996. 